\title{
Influence of the association between simvastatin and demineralized bovine bone matrix on bone repair in rats
}

\section{Carlos Eugênio Villaboim de Castro Lima (a) Jimmy Cavalcanti Calixto(a) Ana Lia Anbinder ${ }^{(b)}$}

(a) Department of Dentistry, UNITAU University of Taubaté, Taubaté, SP, Brazil.

(b) Department of Bioscience and Oral Diagnosis, São José dos Campos School of Dentistry, UNESP - Univ Estadual Paulista, São José dos Campos, SP, Brazil.

\author{
Corresponding author: \\ Ana Lia Anbinder \\ Av. Engenheiro Francisco José Longo, 777, \\ Jardim São Dimas \\ São José dos Campos - SP - Brazil \\ CEP: 12245-000 \\ E-mail: ana.anbinder@fosjc.unesp.br
}

Received for publication on Oct 06, 2010 Accepted for publication on Dec 02, 2010

\begin{abstract}
Simvastatin, a drug used to lower blood cholesterol, has been reported to have an anabolic effect on bone. The purpose of this study was to evaluate the influence of simvastatin and demineralized bovine bone matrix (DBBM) on the repair of rat calvarial defects. Defects of $5 \mathrm{~mm}$ were created in 64 rats, divided into four groups: no local treatment (control); treatment with DBBM (DBBM); treatment with a combination of simvastatin solution $(2.2 \mathrm{mg} / 50 \mu \mathrm{l})$ and DBBM (DBBMSIM-1); and treatment with simvastatin solution $(0.5 \mathrm{mg} / 50 \mu \mathrm{l})$ and DBBM (DBBMSIM-2). Animals were sacrificed on postoperative day 30 or 60 , after which the calvariae were X-rayed and prepared for histomorphometric evaluation. The data were submitted to statistical analysis $(\mathrm{p}<0.05)$. Xrays revealed that, on postoperative day 30 , animals treated with a lower dose of simvastatin presented the lowest bone density, whereas on postoperative day 60 the use of simvastatin, regardless of the dose, resulted in lower density than that observed in control and DBBM group samples. Histomorphometric analysis revealed that, on postoperative day 30, both DBBM and DBBMSIM-1 had a negative impact on bone formation. On postoperative day 60 , none of the combinations tested impaired bone repair. These results showed that the association between DBBM and simvastatin had a negative impact on bone repair.
\end{abstract}

Descriptors: Simvastatin; Bone Regeneration; Bone Matrix.

\section{Introduction}

Autotransplantation is the gold standard for the repair of large bone defects. However, this technique has two major disadvantages: donorsite morbidity and bone resorption. In the 1960s, Urist ${ }^{1}$ reported that demineralized bone implants in extraosseous sites induced local mineralization, a process in which mesenchymal cells differentiate and form new bone. Since then, there have been various studies investigating alternatives to autotransplantation, including the use of demineralized bone matrix for bone repair.

Statins are widely used to lower blood cholesterol levels. Some studies have reported that statins can stimulate bone formation by stimulating the production of bone morphogenetic protein- $2 .^{2}$

Statins target the liver and have reduced affinity for bone tissue; in addition, orally administered statins are poorly distributed to bone. ${ }^{3}$ The doses required for statins to have an effect on bone are much higher than 
those required to reduce cholesterol levels and are associated with unacceptable toxicity. ${ }^{3}$ The major limitation to the clinical use of statins for bone regeneration is the lack of an effective carrier for the drug, ${ }^{4}$ since the success of in vivo bone formation depends on high local concentrations of statins. ${ }^{5}$ Various authors have investigated new methods and carriers for the administration of statins:

- local delivery in methylcellulose gel;6,7

- local delivery in polylactic acid/polyglycolic acid copolymer; ${ }^{5}$

- local delivery in gelatin sponge ${ }^{8}$ and

- statins used in combination with calcium sulfate. ${ }^{9,10}$

The results have often been encouraging.

The purpose of the present study was to evaluate the influence of the association between simvastatin and demineralized bovine bone matrix (DBBM) on the repair of rat calvarial defects.

\section{Material and Methods}

A total of 64 adult male Wistar rats (Rattus norvegicus) aged 90 days were used. In order to create the defects, the animals were anesthetized with $13 \mathrm{mg} / \mathrm{kg}$ of xylazine (Anasedan-Vetbrands-Sespo, Jacareí, SP, Brazil) and $33 \mathrm{mg} / \mathrm{kg}$ of ketamine (Dopalen Vetbrands - Sespo, Jacareí, SP, Brazil). An incision was made through the skin and periosteum, and a trephine drill (Neodent, Curitiba, PR, Brazil) was used to create two critical size defects $(5 \mathrm{~mm}$ in diameter), ${ }^{11}$ one in each parietal bone.

The simvastatin solution was prepared by mixing simvastatin (lot no. 0707071512; Galena, Campinas, SP, Brazil) and distilled water at a proportion of $2.2 \mathrm{mg} / 50 \mu \mathrm{l}$ (solution $1^{6}$ ) or of $0.5 \mathrm{mg} / 50 \mu \mathrm{l}$ (solution $2^{7}$ ) and centrifuging the mixture for $1 \mathrm{~h}$. The animals were divided into four groups:

- control, in which the defects were not filled;

- DBBM, in which the defects were filled with $10 \mathrm{mg}$ of DBBM (GenOx Org Cortical Micro; Baumer S.A., Mogi Mirim, SP, Brazil) and $56 \mu 1$ of distilled water;

- DBBMSIM-1, in which the defects were filled with $10 \mathrm{mg}$ of DBBM and $56 \mu$ l of simvastatin solution 1 (Figure 1); and

- DBBMSIM-2, in which the defects were filled

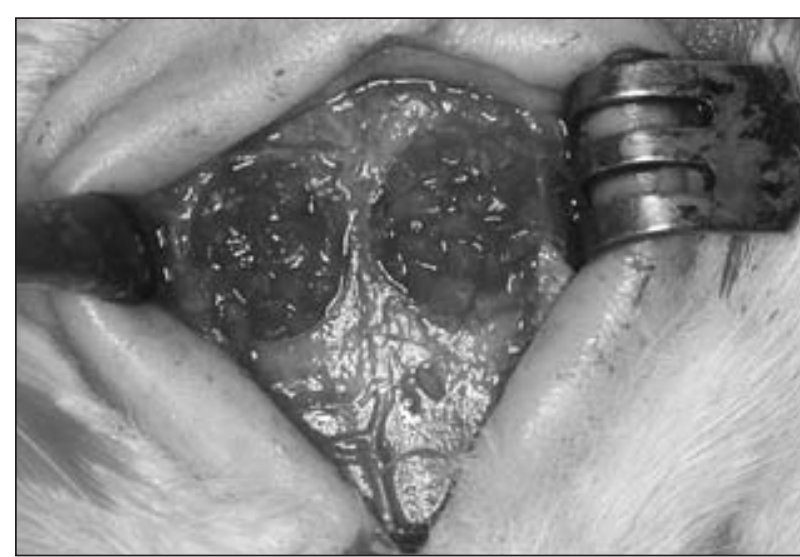

Figure 1 - Defects filled with demineralized bovine bone matrix in combination with simvastatin solution 1 .

with $10 \mathrm{mg}$ of DBBM and $56 \mu \mathrm{l}$ of simvastatin solution 2 .

After 30 or 60 days, the animals were sacrificed, and the calvariae were removed and fixed. After radiographic examination, right and left defects were separated, and left-side samples were sent for decalcification. Right-side samples were embedded in methyl methacrylate resin, cut into sections, and mounted on slides for microscopic examination (data not shown).

The samples were X-rayed using dental X-ray equipment (Spectro 70X; Dabi Atlante, São Paulo, SP, Brazil) with a digital sensor (Trophy RVG, Kodak Dental Systems, Rochester, NY, USA), under the following conditions: $8 \mathrm{~mA} ; 70 \mathrm{KVp}$; focus-object distance of $30 \mathrm{~cm}$; exposure time of $0.1 \mathrm{~s}$. The radiographic density (gray levels) of the region of interest (circular area $=15,380$ pixels) was calculated using the ImageJ 1.31p software (National Institutes of Health, Bethesda, MD, USA). For statistical analysis, we considered the mean density of the two defects for each animal.

Left-side samples were decalcified with 5\% trichloroacetic acid. Semiserial sections of 5-7 $\mu \mathrm{m}$ were cut and stained with hematoxylin and eosin (H\&E). For histomorphometric analysis, the images of four sections were digitized (Axiophot 2 Carl Zeiss, Göttingen, Niedersachsen, Germany) at a magnification of $\times 25$. The Adobe Photoshop software (Adobe Systems Incorporated, San Jose, CA, USA) was used to select areas of newly formed bone, including osteo- 
cyte lacunae. Subsequently, image binarization was performed, and the area was calculated using the ImageJ $1.31 \mathrm{p}$ software.

In order to evaluate the effect of the different types of treatment at each time point $(30$ or 60 days), the densitometric data were submitted to analysis of variance (ANOVA) and Tukey's test, whereas the histomorphometric data were submitted to the Kruskal-Wallis test, followed by Dunn's post-test. In order to evaluate the effect of time, the Student's t-test or the Mann-Whitney test was used. The level of significance was set at $5 \%$ for all tests. The analyses were performed with GraphPad Prism 4.0 (GraphPad Softwares Inc., San Diego, CA, USA) and Bioestat 2.0 (Sociedade Civil Mamirauá, Belém, PA, Brazil).

The present study was approved by the Animal Research Ethics Committee of the University of Taubaté, Brazil (protocol no. 0016/07).

\section{Results}

After the surgical procedure, 6 rats from group DBBMSIM-1 presented scabbing - which persisted for 30 days - at the incision site. Only 1 animal from group DBBMSIM-2 presented scabbing, observed 14 days after surgery. The lesion was approximately half the size of that seen in animals from group DBBMSIM-1 and had disappeared on postoperative day 30. Also in group DBBMSIM-2, 1 animal died from anesthesia-related complications and was not replaced.

\section{X-ray densitometry}

The ANOVA revealed a significant difference between the radiographic density values of the samples collected on postoperative day $30(\mathrm{~F}=3.395, \mathrm{df}=3$, $\mathrm{p}=0.0316)$ and those of the samples collected on postoperative day $60(\mathrm{~F}=8.574, \mathrm{df}=3, \mathrm{p}=0.0004)$. Figures 2 and 3 illustrate the differences revealed by Tukey's test.

The Student's t-test, used to analyze the effects of time on radiographic density according to the type of treatment, revealed no significant differences between the two time points in group DBBMSIM-1 (Figure 4). In the remaining groups, significantly greater radiographic density was observed in ani-

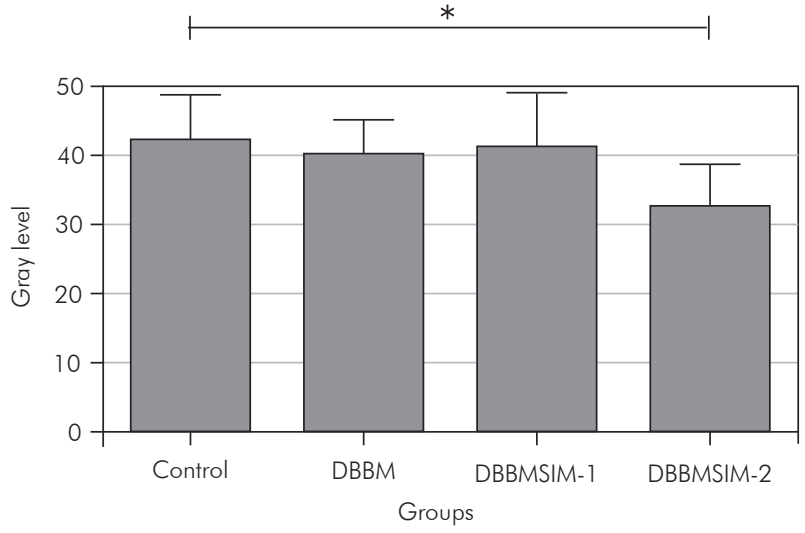

Figure 2 - Means and standard deviations of radiographic density values on postoperative day $30 .{ }^{*}$ indicates significant difference (Tukey's test).

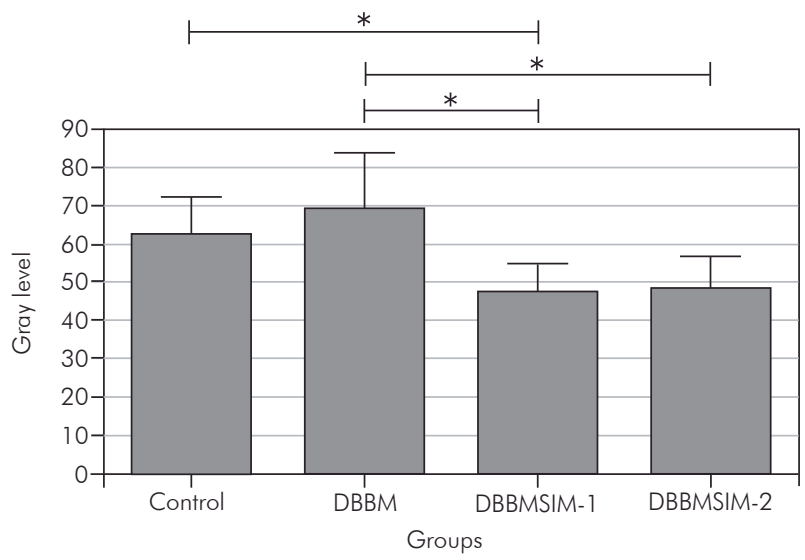

Figure 3 - Means and standard deviations of radiographic density values on postoperative day $60 .{ }^{*}$ indicates significant difference (Tukey's test).

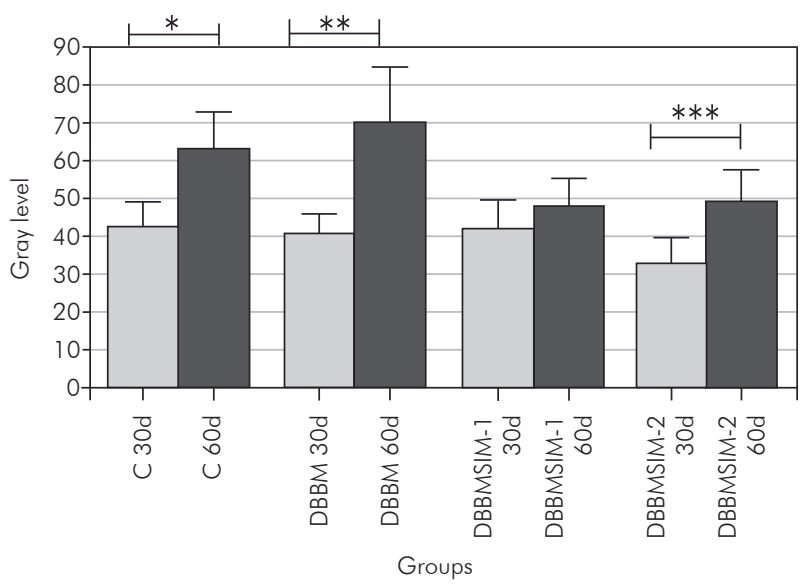

Figure 4 - Means and standard deviations of radiographic density values on postoperative days 30 and $60 .{ }^{*} \dagger=5.024$, $\mathrm{df}=14, \mathrm{p}=0.0002 ;^{* *} \mathrm{t}=5.348, \mathrm{df}=14, \mathrm{p}=0.0001$; $* * * t=4.249, \mathrm{df}=13, \mathrm{p}=0.0009$. 


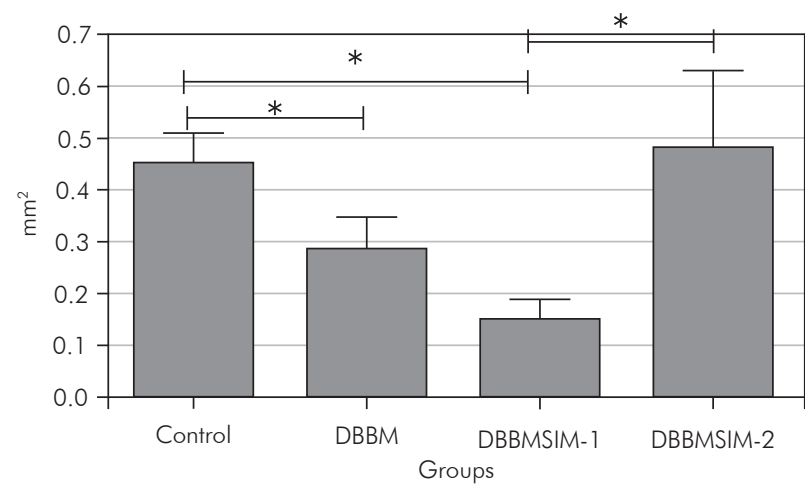

Figure 5 - Means and standard errors of new bone formation values on postoperative day $30 .{ }^{*}$ indicates significant difference (Dunn's post-test).

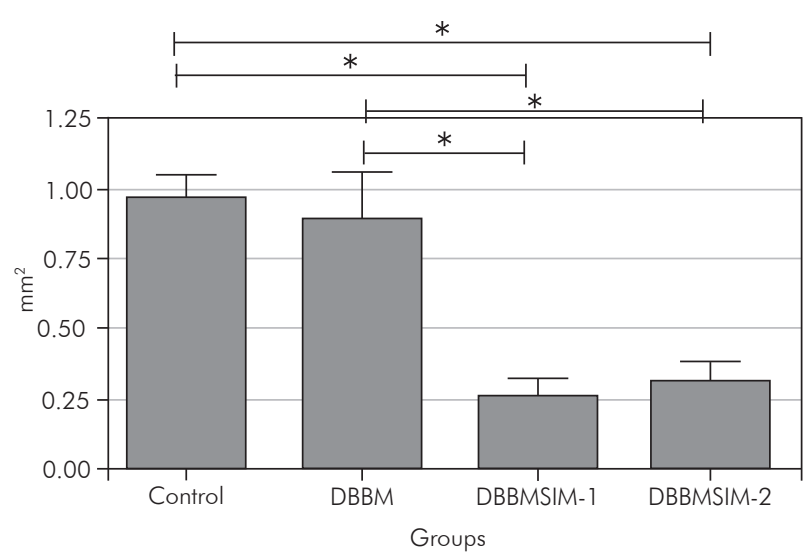

Figure 6 - Means and standard errors of new bone formation values on postoperative day $60 .{ }^{*}$ indicates significant difference (Dunn's post-test).

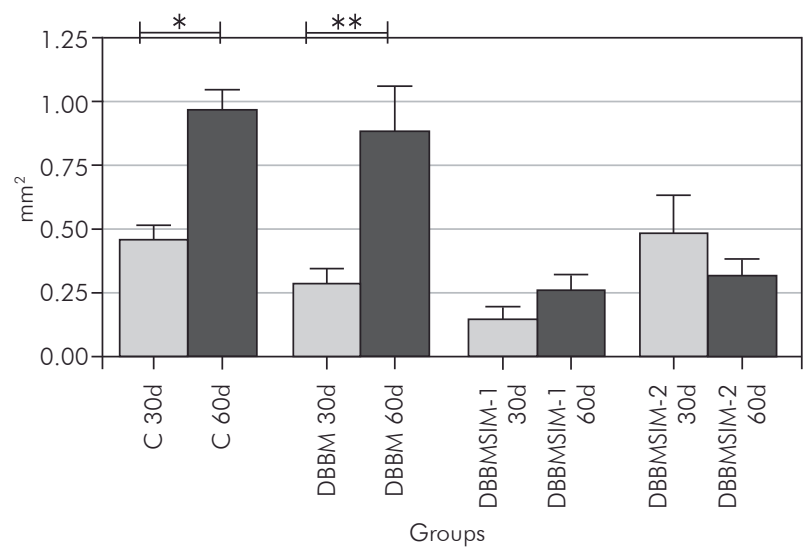

Figure 7 - Means and standard errors of new bone formation values on postoperative days 30 and $60 .{ }^{*} t=5.36$, $\mathrm{df}=10.29, \mathrm{p}=0.0003 ;^{* *} \dagger=3.3311, \mathrm{df}=8.86$, $\mathrm{p}=0.0103$. mals sacrificed on postoperative day 60 .

\section{Histomorphometric analysis}

Due to technical difficulties during decalcification and slide preparation, one sample collected from group DBBM on postoperative day 30 was not used. The Kruskal-Wallis test revealed a significant difference between the types of treatment, both on postoperative day $30(\mathrm{H}=13.592$; $\mathrm{df}=3 ; \mathrm{p}=0.0035)$ and on postoperative day 60 $(\mathrm{H}=16.828 ; \mathrm{df}=3 ; \mathrm{p}=0.0008)$. Dunn's post-test revealed that, on postoperative day 30 , both DBBM and DBBMSIM-1 had a negative influence on new bone formation (Figure 5). On postoperative day 60, the two different doses of simvastatin had a negative influence on bone repair (Figure 6).

The effect of time on bone repair was significant only in animals from the control group and in those from group DBBM, the highest density values being observed on postoperative day 60 (Figure 7).

\section{Descriptive histology}

On postoperative day 30 , the examination of control samples revealed an area of new bone at the edges of the defects. The newly formed bone presented plump osteocytes arranged in a disorganized manner and was covered by flattened osteoblasts. Small bone islands were seen in the center of the defect, which was filled with loose connective tissue and mild chronic inflammatory infiltrate. The histological features of group DBBM samples were similar to those of control samples; however, there was less new bone at the edges and in the center of the defect. The connective tissue was thicker in some samples and presented moderate chronic inflammatory infiltrate. DBBM had been completely reabsorbed in all but 1 of the animals from group DBBM. Group DBBMSIM-1 samples presented few areas of new bone formation, limited to the edges of the defect and composed of highly immature tissue surrounded by plump osteoblasts (Figure 8), as well as regions of old bone resorption. The connective tissue that filled the defect was highly vascularized, thicker than that observed in groups control and DBBM, and presented moderate or severe chronic 


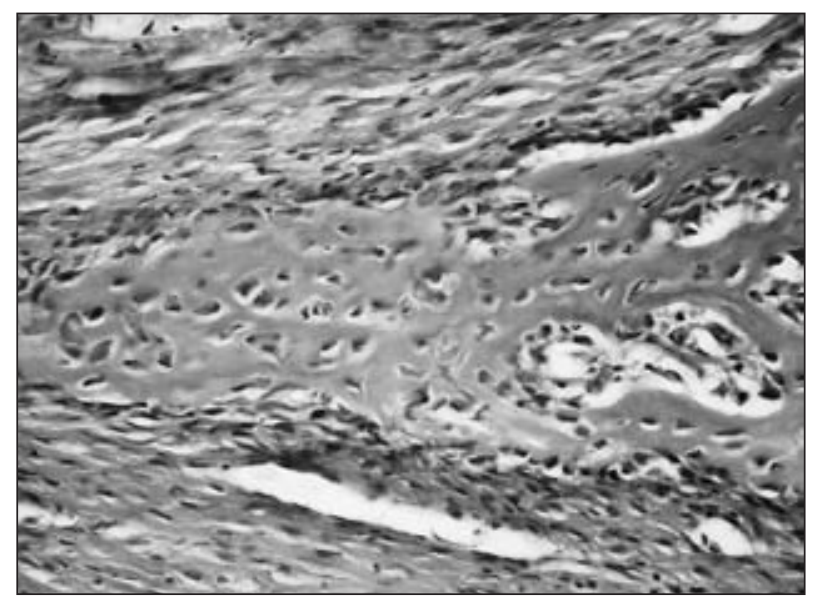

Figure 8 - Microscopic appearance of a sample from an animal from group DBBMSIM-1 that was sacrificed on postoperative day 30 . Very immature bone tissue, with plump osteocytes and osteoblasts (H\&E; ×400).

inflammation. An area of necrosis was observed in 1 animal, and remnants of DBBM were seen amid connective tissue in $50 \%$ of group DBBMSIM- 1 animals. Group DBBMSIM-2 samples presented few areas of new bone formation, with highly immature tissue. However, there was no resorption of old bone. Remnants of DBBM amid the connective tissue that filled the defect were observed in 1 animal. No areas of necrosis were observed.

On postoperative day 60, the newly formed bone at the edges of the defect in control samples was thicker and more mature, containing osteocytes that were smaller and more regularly arranged than those observed on postoperative day 30. In a large number of samples, we observed islands of newly formed bone in the center of the defect. In some samples, linear closure of the defect was nearly complete. Areas of mild inflammation were still present. The histological features of group DBBM samples were similar to those of control samples. DBBMSIM-1 samples once again presented little new bone formation (Figure 9). The connective tissue was slightly thicker and more vascularized than that observed in groups control and DBBM, presenting mild or moderate inflammation. No bone formation was observed in the center of the defect in any of the animals from group DBBMSIM-1. Osteoblasts and osteocytes were plumper in group DBBMSIM-1

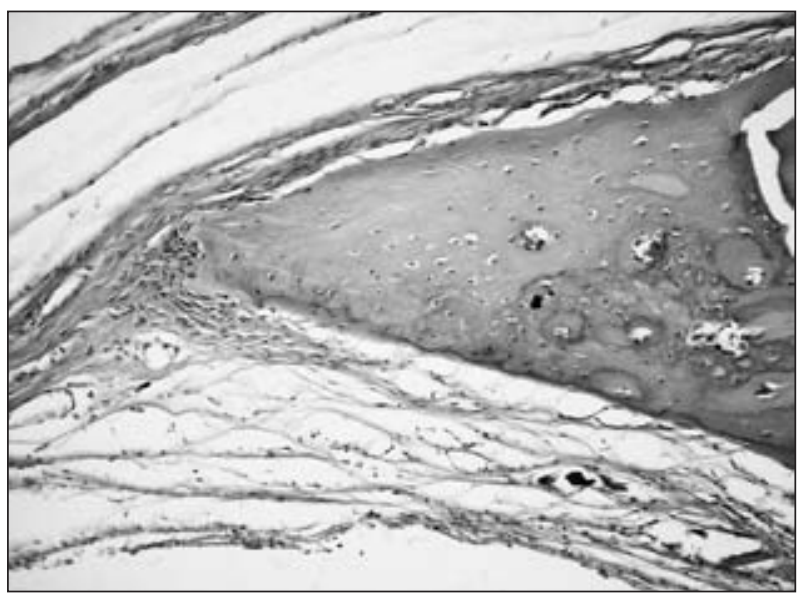

Figure 9 - Microscopic appearance of a sample from an animal from group DBBMSIM-1 that was sacrificed on postoperative day 60. Small amount of bone formation at the edges of the defect $(H \& E ; \times 200)$.

samples than in the samples from the remaining groups, and remnants of DBBM were seen in 3 animals. Group DBBMSIM-2 samples once again presented little bone formation. The connective tissue that filled the defect was thin, presenting mild chronic inflammatory infiltrate. Neither remnants of DBBM nor necrosis was observed in any of the animals from group DBBMSIM-2.

\section{Discussion}

A critical size defect can be defined as the smallest size of an intraosseous wound - in a particular bone and species of animal - that will not heal spontaneously during the lifetime of the animal ${ }^{12}$ or over the duration of the study. ${ }^{13} \mathrm{~A} 5-\mathrm{mm}$ rat calvarial defect is considered a critical size defect. ${ }^{11}$ Our analysis of cross-sectional area revealed that none of the samples from the control group presented complete bone regeneration, which allowed us to analyze the osteogenic potential of the treatments tested.

In the present study, X-ray densitometry and histomorphometric analysis were complementary because densitometry was used to evaluate calcified tissue and histomorphometric analysis was used to evaluate decalcified tissue. Although radiographic analysis has certain advantages, such as low cost and rapid results, the accuracy of the method when employed in rat calvarial defect models has been re- 
ported to be low. ${ }^{14}$ Therefore, radiographic analysis should not be used as the only method of evaluation (i.e., without histological analysis, considered the gold standard). Our results confirmed this. On postoperative day 30, radiographic and histological analyses revealed opposite results for group DBBMSIM-2. On postoperative day 60, when bone formation was more evident, the two analyses revealed no conflicting results.

Although DBBM has osteoconductive ${ }^{15}$ and osteoinductive properties, osteoinductivity varies according to the commercial preparation. ${ }^{16} \mathrm{We}$ were unable to confirm the beneficial properties of DBBM..$^{15,17}$ Carneiro et al. ${ }^{18}$ also investigated the beneficial effects of DBBM and reported no significant differences between the treatment group and the control group. In the present study, DBBM delayed bone matrix formation in the first 30 days, exacerbating the inflammatory process. A more intense initial inflammation might be related to factors such as incomplete decalcification of the graft, residual lipids or immunopathological features of the recipient..$^{18}$ In the present study, the association between inflammation and DBBM resorption in the first 30 days might have affected the osteoconductivity of DBBM. On postoperative day 60, when DBBM was no longer present, no significant differences were observed between groups DBBM and control.

Various authors have investigated local delivery of statins to increase bone formation and reported that statins have a local anabolic effect. ${ }^{5,9,10}$ Others, however, have found no beneficial effects. ${ }^{9,19,20}$ The ideal doses and carriers have yet to be determined, and the issue remains controversial. Low doses have no impact on bone repair, whereas high doses can stimulate inflammation. High local concentrations of statins can be cytotoxic due to a drastic reduction in the production of cholesterol, a substance that is important to the integrity of cell membranes. ${ }^{4}$

In the present study, the use of high local doses of simvastatin caused an intense inflammatory reaction, accompanied by tissue necrosis and scabbing. Soft tissue inflammation was also reported by Thylin et al., ${ }^{6}$ who used $2.2 \mathrm{mg}$ of the drug in methylcel- lulose gel in rat calvaria; by Nyan et al., ${ }^{9}$ who used $1 \mathrm{mg}$ of the drug in calcium sulfate in rat calvaria; and by Stein et al., ${ }^{7}$ who used doses of $0.5,1.0,1.5$, and $2.2 \mathrm{mg}$ of the drug in rat mandible. In the study conducted by Stein et al., ${ }^{7}$ the 0.5 -mg dose presented the best cost-benefit ratio, promoting significant bone formation and reduced inflammation. Sato et al.,${ }^{21}$ however, used $2 \mathrm{mg}$ of simvastatin in calcium sulfate in rat alveolar bone after incisor extraction and reported no adverse effects. The speed at which the carrier releases the drug also plays a role in the local concentration and effectiveness of simvastatin. Stein et al. ${ }^{7}$ used $0.1 \mathrm{mg}$ of simvastatin in methylcellulose gel and reported no stimulation of bone formation, whereas Nyan et al. ${ }^{10}$ used the same amount of simvastatin in combination with alpha-tricalcium phosphate and reported significant new bone formation, greater than that obtained using 0.25 and $0.5 \mathrm{mg}$ of the drug.

In the present study, DBBM might not have been an effective carrier of the aqueous solution of simvastatin, since the drug delayed DBBM resorption, prolonging the inflammation process. The effects of the association between DBBM and a higher dose of simvastatin were already evident on postoperative day 30, when decreased bone formation was observed in group DBBMSIM-1, whereas the effects of the association between DBBM and a lower dose of simvastatin (group DBBMSIM-2) were only evident on postoperative day 60. Despite the evident reduction in inflammation in the DBBMSIM-2 group, the two doses used delayed bone repair. In a study conducted by $\mathrm{Ma}$ et al., ${ }^{20}$ local delivery of $0.1,0.9$, or $1.7 \mathrm{mg}$ of simvastatin to tibia implants in combination with beta-tricalcium phosphate also had a negative effect on bone repair. Further studies aiming at determining optimal doses and routes of administration of statins are required in order to evaluate more accurately the use of these drugs for bone repair.

\section{Conclusion}

The results of the present study showed that the association between DBBM and simvastatin had a negative impact on bone repair, and that none of the 
treatments tested improved bone regeneration.

\section{Acknowledgements}

This research was supported by FAPESP, Grant

\section{References}

1. Urist MR. Bone: formation by autoinduction. Science. 1965 Nov 12;150 (3698):893-9.

2. Mundy G, Garrett R, Harris S, Chan J, Chen D, Rossini G, et al. Stimulation of bone formation in vitro and in rodents by statins. Science. 1999 Dec 3;286(5446):1946-9.

3. Gutierrez GE, Lalka D, Garrett IR, Rossini G, Mundy GR. Transdermal application of lovastatin to rats causes profound increases in bone and plasma concentrations. Osteoporos Int. 2006;17(7):1033-42.

4. Benoit DS, Nuttelman CR, Collins SD, Anseth KS. Synthesis and characterization of a fluvastatin- releasing hydrogel delivery system to modulate hMSC differentiation and fuction for bone regeneration. Biomaterials. 2006 Dec;27(36):6102-10.

5. Wu Z, Liu C, Zang G, Sun H. The effect of simvastatin on remodelling of the alveolar bone following tooth extraction. Int J Oral Maxillofac Surg. 2008 Feb;37(2):170-6.

6. Thylin MR, McConnell JC, Schmid MJ, Reckling RR, Ojha $\mathrm{J}$, Bhattacharyya I, et al. Effects of simvastatin gels on murine calvarial bone. J Periodontol. 2002 Oct;73(10):1141-8.

7. Stein D, Lee Y, Schmid MJ, Killpack B, Genrich MA, Narayana $\mathrm{N}$, et al. Local simvastatin effects on mandibular growth and inflammation. J Periodontol. 2005 Nov;76(11):1861-70.

8. Kiliç E, Özeç I, Yeler H, Korkmaz A, Ayas B, Gümüs C. Effects of simvastatin on mandibular distraction osteogenesis. J Oral Maxillofac Surg. 2008 Nov;66(11):2233-8.

9. Nyan M, Sato D, Oda M, Machida T, Kobayashi H, Nakamura $\mathrm{T}$, et al. Bone formation with the combination of simvastatin and calcium sulfate in critical sized rat calvarial defect. J Pharmacol Sci. 2007 Aug;104(4):384-6.

10. Nyan M, Sato D, Kihara H, Machida T, Ohya K, Kasugai S. Effects of the combination with alpha- tricalcium phosphate and simvastatin on bone regeneration. Clin Oral Implants Res. 2009 Mar;20(3):280-7.

11. Bosch C, Melsen B, Vargervik K. Importance of the criticalsize bone defect in testing bone-regenerating materials. J Craniofac Surg. 1998 Jul;9(4):310-6. no. 07/55508-8. We would like to thank Maria de Fátima Pacheco for her aid in preparing the histological slides.

12. Hollinger JO, Kleinschmidt MD. The critical size defect as an experimental model to test bone repair materials. J Craniofac Surg. 1990 Jan;1(1);60-8.

13. Gosain AK, Song L, Yu P, Mehara BJ, Maeda CY, Gold LI, et al. Osteogenesis in cranial defects: reassesment of the concept of critical size and the expression of TGF-(beta) isoforms. Plast Reconstr Surg. 2000 Aug;106(2);360-72.

14. Pryor ME, Susin C, Wikesjö UM. Validity of radiographic evaluations of bone formation in a rat calvaria osteotomy defect model. J Clin Periodontol. 2006 Jun;33(6):455-60.

15. Herculiani PP, Cestari TM, Taga EM, Taga R. [Treatment of perennial bone defect in guinea pigs skull freeze dryed associated or no to bovine bone graft demineralized]. Rev Bras Implant. 2000 Abr-Jun;6(2):7-14

16. Wang JC, Alanay A, Mark D, Kanim LE, Campbell PA, Dawson EG, et al. A comparison of commercially available demineralized bone matrix for spinal fusion. Eur Spine J. 2007 Aug;16(8):1233-40.

17. Marins LV, Cestari TM, Sottovia AD, Granjeiro JM, Taga R. Radiographic and histological study of perennial bone defect repair in rat calvaria after treatment with blocks of porous bovine organic graft material. J Appl Oral Sci. 2004 JanMar;12(1):62-9.

18. Carneiro E, Garcia RB, de Oliveira RC, de Moraes FG, Menezes R, Letra A, et al. Microscopic and radiographic analysis of the effect of particle size of demineralized bovine cancellous bone matrix on the repair of bone defects in femurs of rabbits. J Appl Oral Sci. 2005 Apr-Jun;13(2):157-62.

19. Von Stechow D, Fish S, Yahalom D, Bab I, Chorev M, Müller $\mathrm{R}$, et al. Does simvastatin stimulate bone formation in vivo? BMC Musculoskelet Disord. 2003 Apr 28;4:8.

20. Ma B, Clarke SA, Brooks RA, Rushton N. The effect of simvastatin on bone formation and ceramic resorption in a periimplant defect model. Acta Biomater. 2008 Jan;4(1):149-55.

21. Sato D, Nishimura K, Ishioka T, Hondo H, Kuroda S, Kasugai S. Local application of simvastatin to rat incisor socket: carrier-dependent effect on bone augmentation. J Oral Tissue Engin. 2005;2(2):81-5. 